\title{
SOME RESULTS BY USING CLR'S-PROPERTY IN PROBABILISTIC 2-METRIC SPACE
}

\author{
V. SRINIVAS ${ }^{1}$, K. SATYANNA2,* \\ ${ }^{1}$ Department of Mathematics, University College of Science, Osmania University, Hyderabad, Telangana \\ State, India \\ 2Department of Mathematics, M.A.L.D. Government Degree College, Gadwal, Palamoor University, \\ Mahaboobnagar, Telangana State, India \\ *Corresponding author: satgjls@gmail.com
}

ABSTRACT. The aim of this paper is to generate two fixed point theorems in probabilistic 2-metric space by applying CLR's-property and occasionally weakly compatible mappings (OWC), these two results generalize the theorem proved by V. K. Gupta, Arihant Jain and Rajesh Kumar. Further these results are justified with suitable examples.

\section{INTRODUCTION}

Menger [1] pioneered the statistical metric(SM) space theory. One of the major achievements was the translation of probabilistic concepts into geometry. Menger used the notation of new distance distribution function from $\mathrm{p}$ to $\mathrm{q}$ by a Fpq. B. Schweizer, and A. Sklar [2] introduced a new notion of a probabilistic-norm. This norm naturally generates topology, convergence, continuity and completeness in SM-space. Mishra [3] used compatible mappings and generated some fixed points in Menger space. Altumn Turkoglu [4] proved some more results of SM-space by utilizing the implicit relation in multivalued mappings. Zhang, Xiaohong, Huacan He, and Yang Xu [5] employed the Schweizer-Sklar t-norm established fuzzy

Received August 15th, 2021; accepted October 4th, 2021; published November $3^{\text {rd }}, 2021$.

2010 Mathematics Subject Classification. 54H25, 47H10.

Key words and phrases. self-mappings; occasionally weakly compatible mappings; probabilistic 2-metric space; CLR's-property.

C2021 Authors retain the copyrights of their papers, and all open access articles are distributed under the terms of the Creative Commons Attribution License. 
logic system to contribute in development of SM-space. Sehgal, V. M., and A. T. Bharucha-Reid [6] used classical Banach contraction to establish the first result of Menger space for coincidence points. Weakly compatible mappings were generalized by Al-Thagafi and Shahzad [7], by introducing occasionally weakly compatible mappings. Futher Chauhan, Sunny, Wutiphol Sintunavarat, and Poom Kumam[9] proved some more theorems by using CLR'S-property in fuzzy metric space. Further some more results can be witnessed by using the concepts of sub sequentially continuous and semi compatible mappings in Menger space [10].

\section{PRELIMINARIES}

Definition 2.1 [8] A continuously t-norm is mapping t: $[0,1] \times[0,1] \rightarrow[0,1]$ and it satisfies the following properties

$\left(t_{1}\right) t$ is abelian \& associative

$\left(t_{2}\right) t(\gamma, 1)=\gamma, \forall \gamma \in[0,1]$

$\left(t_{3}\right) t(\gamma, \omega) \leq t(\alpha, \vartheta)$ for $\gamma \leq \alpha$ and $\omega \leq \vartheta \forall \gamma, \alpha, \vartheta, \omega \in[0,1]$.

Definition 2.2 [8] The pair $(X, F)$ named as Probabilistic 2-metric space (2-PM space) where $X \neq$ $\emptyset$ and $F: X \times X \times X \rightarrow L$ here $L$ is the set of all distribution functions and the $F$ value at $(u, v, w) \in X \times X \times X$ is represented by $F_{u, v, w}$ and obeys properties as under

a) $F_{u, v, w}(0)=0$,

b) For all distinct $\mathrm{u}, \mathrm{v}$ in $\mathrm{X} \exists \mathrm{w} \in \mathrm{X}$ with $F_{u, v, w}(\mathrm{t})<1$ for some $\mathrm{t}>0$,

c) $F_{u, v, w}(\mathrm{t})=1 \forall \mathrm{t}>0$, If any two of the three points have to be the same,

d) $F_{u, v, w}(\mathrm{t})=F_{v, w, u}(\mathrm{t})=F_{w, u, v}(\mathrm{t})$,

e) $F_{u, v, w}\left(t_{a}\right)=F_{v, w, u}\left(t_{b}\right)=F_{w, u, v}\left(t_{c}\right)=1$ then $F_{u, v, w}\left(t_{a}+t_{b}+t_{c}\right)=1$.

Definition 2.3 [8] A sequence $\left\langle x_{n}\right\rangle$ in 2-Menger space $(X, F, t)$ is

(i) Converges to $\beta$ if for each $\epsilon^{*}>0, t>0, \exists \mathrm{N}\left(\epsilon^{*}\right) \in \mathrm{N}$ implies $F_{x_{n}, \beta, a}\left(\epsilon^{*}\right)>1-\mathrm{t}$, $\forall$ a $\mathrm{X}$ and $n \geq N\left(\epsilon^{*}\right)$.

(ii) Cauchy if for each $\epsilon>0, t>0, \exists \mathrm{N}(\epsilon) \in \mathrm{N}$ implies $F_{x_{n}, x_{m}, a}(\epsilon)>1-\mathrm{t}$, $\forall a \in X$ and $n, m \geq N(\epsilon)$

(iii) If the cauchy sequence converges in $X$ then it is referred as a complete 2-Menger space. 
Definition 2.4 [8] Self-mappings $\mathrm{P}, \mathrm{S}$ in 2-Menger space $(X, F, t)$ are called

(i) Compatible If $F_{P S x_{n}, S P x_{n}, a}(\delta) \rightarrow 1, \forall a \in X$ and $\delta>0$ whenever a sequence $\left\langle x_{n}\right\rangle$ in $X$ $\exists P x_{n}, S x_{n} \rightarrow Z$ where $\mathrm{z}$ is an element of $\mathrm{X}$ as $\mathrm{n} \rightarrow \infty$.

(ii) Weakly compatible if the mappings commute at their coincidence points.

(iii) Occasionally weakly compatible (OWC) if $\exists \mathrm{x}$ in $\mathrm{X} \ni \mathrm{Px}=\mathrm{Sx} \Rightarrow \mathrm{PSx}=\mathrm{SPx}$.

Remark 2.5 Two weakly compatible mappings are obviously OWC mappings, but the converse does not have to be the case.

Example 2.6. By treating $X=[0,1]$ and $d$ be the usual metric on $X$ and for all $t_{1} \in[0,1]$, define

$F_{u, v, a}\left(t_{1}\right)=\left\{\begin{array}{cl}\frac{t_{1}}{t_{1}+|\alpha-\beta|}, & t_{1}>0 \\ 0, & t_{1}=0\end{array} \quad \forall \alpha, \beta\right.$ in $\mathrm{X}$ and fixed $\mathrm{a}, t_{1}>0$.

Define mappings $P, S: X \rightarrow X$ as $P(x)=\frac{x^{2}}{2}, \mathrm{x} \in[0,1]$ and $S(x)=\frac{x}{3^{\prime}} \mathrm{x} \in[0,1]$.

We notice that the pair $(\mathrm{P}, \mathrm{S})$ has two coincidence points $0, \frac{2}{3}$.

If $x=\frac{2}{3}$ then $P\left(\frac{2}{3}\right)=S\left(\frac{2}{3}\right)=\frac{2}{9}$

$P S\left(\frac{2}{3}\right)=P\left(\frac{2}{9}\right)=\frac{2}{81}$

$S P\left(\frac{2}{3}\right)=S\left(\frac{2}{9}\right)=\frac{2}{27}$.

From (2.6.2) and (2.6.3) $\mathrm{PS}\left(\frac{2}{3}\right) \neq \mathrm{SP}\left(\frac{2}{3}\right)$.

At $\mathrm{x}=0, \mathrm{P}(0)=\mathrm{S}(0)$ and $P S(0)=S P(0)$.

This shows the mappings $\mathrm{P}, \mathrm{S}$ are OWC but not weakly compatible.

Definition 2.7 [9] “Self maps $\mathrm{P}$ and $\mathrm{S}$ of a 2-Menger space $(X, F, t)$ are said to satisfy $\mathrm{CLR}_{\mathrm{S}}$ - property (common limit range property) if there exists a sequence $\left\langle x_{n}\right\rangle \in X \ni P x_{n}, S x_{n} \rightarrow S z$, for some element $\mathrm{z} \in X$ as $\mathrm{n} \rightarrow \infty$.

This example shows that mappings $\mathrm{P}, \mathrm{S}$ satisfy $\mathrm{CLR}_{\mathrm{S}^{-}}$property but they do not have closed ranges.

Example 2.8. Take $X=(0,1]$ and $t \in[0,1]$, define 
$F_{u, v}(\mathrm{t})=\left\{\begin{array}{cl}\frac{t}{t+|\alpha-\beta|}, & t>0 \\ 0, & t=0\end{array} \forall \alpha, \beta\right.$ in $\mathrm{X}$ and $t>0$.

Define $P, S: X \rightarrow X$ as $P(x)=\left\{\begin{array}{r}1-x, x \in\left(0, \frac{2}{3}\right) \\ x, x \in\left[\frac{2}{3}, 1\right]\end{array}\right.$

and

$$
S(x)=\left\{\begin{aligned}
2 x, & x \in\left(0, \frac{2}{3}\right] \\
1, & x \in\left(\frac{2}{3}, 1\right] .
\end{aligned}\right.
$$

Consider a sequence $x_{n}=\frac{1}{3}-\frac{1}{3 n}$ for $\mathrm{n}=1,2,3 \ldots$ then

$\mathrm{P} x_{n}=1-\left(\frac{1}{3}-\frac{1}{3 n}\right)=\frac{2}{3}+\frac{1}{3 n} \rightarrow \frac{2}{3}$

$\mathrm{S} x_{n}=2\left(\frac{1}{3}-\frac{1}{3 n}\right)=\frac{2}{3}-\frac{2}{9 n} \rightarrow \frac{2}{3}$ as $\mathrm{n} \rightarrow \infty$.

Thus $P x_{n}, S x_{n} \rightarrow S\left(\frac{1}{3}\right)=\frac{2}{3}$ as $\mathrm{n} \rightarrow \infty$.

Where $P(X)=\left(\frac{1}{3}, 1\right], S(X)=\left(0, \frac{4}{3}\right] U\{1\}$ this shows that $P, S$ are satisfy CLR' $S^{-}$property but they do not have closed ranges.

Now we give the statement of Theorem (A). It is proved by V. K. Gupta et al.

Theorem (A) [8] “ Let A, B, S and T be self -mappings on a complete probabilistic 2-metric space $(\widetilde{\mathrm{X}}, \mathrm{F}, \mathrm{t})$ satisfying:

$\left(\mathrm{A}_{1}\right) \mathrm{A}(\widetilde{\mathrm{X}}) \subseteq \mathrm{T}(\widetilde{\mathrm{X}}), \mathrm{B}(\widetilde{\mathrm{X}}) \subseteq \mathrm{S}(\widetilde{\mathrm{X}})$

$\left(A_{2}\right)$ one of $\mathrm{A}(\widetilde{\mathrm{X}}), \mathrm{B}(\widetilde{\mathrm{X}}), \mathrm{T}(\widetilde{\mathrm{X}})$ or $\mathrm{S}(\widetilde{\mathrm{X}})$ is complete,

$\left(A_{3}\right)$ pairs $(\mathrm{A}, \mathrm{S})$ and $(\mathrm{B}, \mathrm{T})$ are weakly compatible,

$\left(A_{4}\right) F_{A x . B y, a}(\mathrm{t}) \geq \mathrm{r} F_{S x} . T y, a(\mathrm{t})$ for all $\mathrm{x}, \mathrm{y}$ and $\mathrm{t}>0$,

where $r:[0,1] \rightarrow[0,1]$ is some continuous function such that $r(t)>t$ for each $o<t<1$, then $\mathrm{A}, \mathrm{B}, \mathrm{S}$ and $\mathrm{T}$ have unique common fixed point in $\widetilde{\mathrm{X}}^{\prime \prime}$.

We now generalize Theorem(A) as under.

\section{MAIN RESULT}

Theorem 3.1 Let A, B, S and T be self -mappings on a complete probabilistic 2-metric space $\left(\widetilde{\mathrm{X}}, \mathrm{F}, t^{*}\right)$ satisfying :

(3.1.1) $\mathrm{A}(\widetilde{\mathrm{X}}) \subseteq \mathrm{T}(\widetilde{\mathrm{X}}), \mathrm{B}(\widetilde{\mathrm{X}}) \subseteq \mathrm{S}(\widetilde{\mathrm{X}})$,

(3.1.2) the pairs $(A, S),(B, T)$ share the $C L R^{\prime}$ s property with $\mathrm{OWC}$, 
(3.1.3) $F_{A x . B y, a}\left(t^{*}\right) \geq \mathrm{r} F_{S x . T y, a}\left(t^{*}\right)$ for all $\mathrm{x}, \mathrm{y}$ and $t^{*}>0$,

where $\mathrm{r}:[0,1] \rightarrow[0,1]$ is some continuous function such that $\mathrm{r}\left(t^{*}\right)>t^{*}$ for each $\mathrm{o}<t^{*}<1$

then $\mathrm{A}, \mathrm{B}, \mathrm{S}$ and $\mathrm{T}$ have unique common fixed point in $\widetilde{\mathrm{X}}$.

\section{Proof:}

Iteratively the sequences $\left\langle y_{n}\right\rangle$ and $\left\langle x_{n}\right\rangle$ can be constructed as

$\mathrm{x}_{0} \in \widetilde{\mathrm{X}} \Rightarrow \mathrm{A} \mathrm{x}_{0} \in \mathrm{A}(\widetilde{\mathrm{X}}) \subseteq \mathrm{T}(\widetilde{\mathrm{X}}), \exists \mathrm{x}_{1} \in \widetilde{\mathrm{X}}$ in such a way that $\mathrm{Ax}_{0}=\mathrm{Tx}_{1}$,

$\mathrm{Bx}_{1} \in \mathrm{B}(\widetilde{\mathrm{X}}) \subseteq \mathrm{S}(\widetilde{\mathrm{X}})$ then we have $\mathrm{x}_{2} \in \widetilde{\mathrm{X}}$ with $\mathrm{Bx}_{1}=\mathrm{Sx}_{2}$

$\left\langle y_{2 n}\right\rangle=\mathrm{A} x_{2 n}=\mathrm{T} x_{2 n+1}$ and $\left\langle y_{2 n+1}\right\rangle=\mathrm{B} x_{2 n+1}=\mathrm{S} x_{2 n+2}$.

Now our claim is to show $\left\langle y_{n}\right\rangle$ is cauchy sequence.

For this take $x=x_{2 n}, y=x_{2 n+1}$ in (3.1.3) we get

$F_{A x_{2 n}, B x_{2 n+1, a}}\left(t^{*}\right) \geq r F_{S x_{2 n} . T x_{2 n+1}}\left(t^{*}\right)$,

$\Rightarrow F_{y_{2 n},} y_{2 n+1, a}\left(t^{*}\right) \geq r F_{y_{2 n-1}, y_{2 n, a}}\left(t^{*}\right)>F_{y_{2 n-1}, y_{2 n, a}}\left(t^{*}\right)$.

Similarly

$F_{y_{2 n+1},} y_{2 n+2, a}\left(t^{*}\right)>F_{y_{2 n}, y_{2 n+1, a}}\left(t^{*}\right)$.

In general we have $F_{y_{n+1},} y_{n}, a\left(t^{*}\right)>F_{y_{n}, y_{n-1, a}}\left(t^{*}\right)$ for all values of $\mathrm{n}$.

Then $<F_{y_{n+1}} \cdot y_{n}, a\left(t^{*}\right)>$ is an increasing sequence bounded above by 1 therefore it must converge to $\mathrm{L}$, where $\mathrm{L} \leq 1$.

If $\mathrm{L}<1$ then $F_{y_{n+1}}, y_{n}, a\left(t^{*}\right)=\mathrm{L}>\mathrm{r}(1)>1$ as a result of the contradiction, $\mathrm{L}=1$.

Hence $F_{y_{n+1}}, y_{n}, a(\mathrm{t})=1$ for all $\mathrm{n}$ and $\mathrm{p}$.

As a result, because Cauchy sequence exists in complete space $X$, it has a limit $z$ in $\widetilde{X}$ and consequently each sub sequence has the same limit $\mathrm{z}$.

That is $\mathrm{Ax}_{2 \mathrm{n}}, \mathrm{Sx}_{2 \mathrm{n}} \rightarrow \mathrm{z}$ and $\mathrm{Bx}_{2 \mathrm{n}+1}, \mathrm{Tx}_{2 \mathrm{n}+1} \rightarrow \mathrm{z}$ as $\mathrm{n} \rightarrow \infty$.

On using CLRs-Property of $(\mathrm{A}, \mathrm{S}),(\mathrm{B}, \mathrm{T})$ implies there are

sequences $\left(a_{n}\right)$ as well as $\left(b_{n}\right)$ in order for

$A a_{n}, S a_{n}, B b_{n}, T b_{n} \rightarrow S \mu$ as $n \rightarrow \infty$ for some $\mu$ in $\widetilde{X}$.

To prove $z=S \mu$ put $x=a_{2 n}, y=x_{2 n+5}$ in (3.1.3) we get

$F_{\mathrm{Aa}_{2 n} . B \mathrm{x}_{2 n+5}, a}\left(t^{*}\right) \geq \mathrm{r}\left(F_{\mathrm{Sa}_{2 n} . T \mathrm{x}_{2 n+5}, a}\left(t^{*}\right)\right)$ as $\mathrm{n} \rightarrow \infty$

$\Rightarrow F_{\mathrm{S} \mu . \mathrm{z}, a}\left(t^{*}\right) \geq \mathrm{r}\left(F_{\mathrm{S} \mu . \mathrm{z}, a}\left(t^{*}\right)\right) .>F_{\mathrm{S} \mu . \mathrm{z}, a}\left(t^{*}\right)$.

Resulting $F_{\mathrm{S} \mu . \mathrm{z}, a}\left(t^{*}\right) .>F_{\mathrm{S} \mu \mathrm{z}, a}\left(t^{*}\right)$

which is a contradiction. Hence $S \mu=z$. 
Claim A $\mu=S \mu$.

Put $\mathrm{x}=\mu, \mathrm{y}=\mathrm{x}_{2 \mathrm{n}+3}$ in (3.1.3) we get

$F_{\mathrm{A} \mu . B \mathrm{x}_{2 n+3}, a}\left(t^{*}\right) \geq \mathrm{r}\left(F_{\mathrm{S} \mu . T \mathrm{x}_{2 n+3}, a}\left(t^{*}\right)\right)$ as $\mathrm{n} \rightarrow \infty$

$\Rightarrow F_{\mathrm{A} \mu . \mathrm{z}, a}\left(t^{*}\right) \geq \mathrm{r}\left(F_{\mathrm{S} \mu \mathrm{z}, a}\left(t^{*}\right)\right) \mathrm{using}(3.1 .13)$

$\Rightarrow F_{\text {A } \mu \text {. z, } a}\left(t^{*}\right) \geq \mathrm{r}\left(F_{\text {z. z, } a}\left(t^{*}\right)\right)=\mathrm{r}(1)=1$.

This results $\mathrm{A} \mu=\mathrm{S} \mu=\mathrm{z}$.

Since the pair $(\mathrm{A}, \mathrm{S})$ obeys OWC resulting

$\mathrm{A} \mu=\mathrm{S} \mu \Rightarrow \mathrm{SA} \mu=\mathrm{AS} \mu$. That is $\mathrm{Az}=\mathrm{Sz}$.

Claim $\mathrm{Az}=\mathrm{z}$.

Substitute $y=x_{2 n+3}, x=z$ in (3.1.3) we have

$F_{\text {Az. } B \mathrm{x}_{2 n+3}, a}\left(t^{*}\right) \geq \mathrm{r} F_{\mathrm{Sz} . T \mathrm{x}_{2 n+3}, a}\left(t^{*}\right)$ letting $\mathrm{n} \rightarrow \infty$.

$\Rightarrow F_{A z, z, \text { a }}\left(t^{*}\right) \geq r F_{S z, z, a}\left(t^{*}\right)$ using (3.1.18)

$\Rightarrow F_{A z, z, \text { a }}\left(t^{*}\right) \geq r F_{A z, z, a}\left(t^{*}\right)>F_{A z, z, a}\left(t^{*}\right)$,

$\Rightarrow F_{A z, z, \mathrm{a}}\left(t^{*}\right)>F_{A z, z, a}\left(t^{*}\right)$.

This is a contradiction. Thus $z=A z$.

Resulting $\mathrm{Az}=\mathrm{Sz}=\mathrm{z}$.

Since $A z \in A(\widetilde{X}) \subseteq T(\widetilde{X})$ then $\exists \rho \in \widetilde{X}$ such that $A z=T \rho$.

Claim z $=\mathrm{B} \rho$.

By employing $\mathrm{x}=\mathrm{x}_{4 \mathrm{n}}, \mathrm{y}=\rho$ of (3.1.3) we obtain

$F_{A x_{2 n}, \text { B } \rho}\left(t^{*}\right) \geq r F_{S x_{2 n} n} . T \rho, a\left(t^{*}\right)$ as $\mathrm{n} \rightarrow \infty$.

From (3.1.22) \& (3.1.23)

$\Rightarrow F_{Z, B \rho}\left(t^{*}\right) \geq r F_{z . T \rho, a}\left(t^{*}\right)=\mathrm{r}(1)=1$.

Thus $\mathrm{z}=\mathrm{B} \rho=\mathrm{T} \rho$.

Since the pair of mappings $(B, T)$ obeys OWC, this results

$\mathrm{B} \rho=\mathrm{T} \rho \Rightarrow \mathrm{BT} \rho=\mathrm{TB} \rho$. That is $\mathrm{Bz}=\mathrm{Tz}$.

Claim $\mathrm{z}=\mathrm{Bz}$.

By substituting $y=z, x=z$ in (3.1.3) results

$F_{A z, B z, \quad \text { a }}\left(t^{*}\right) \geq r F_{S z . T z, a}\left(t^{*}\right)$ using (3.1.22) \&(3.1.26)

$F_{z, \text { Bz, a }}\left(t^{*}\right) \geq r F_{z, B z, a}\left(t^{*}\right)>F_{z, B z, a}\left(t^{*}\right)$.

Resulting $F_{z, \mathrm{~B} z, \text { a }}\left(t^{*}\right)>F_{z}, B z, a\left(t^{*}\right)$. It is impossible. Therefore $\mathrm{Bz}=\mathrm{z}$.

Combining all we get $\mathrm{Az}=\mathrm{Bz}=\mathrm{z}=\mathrm{Sz}=\mathrm{Tz}$. 
Thus $z$ is the required common fixed point for these mappings $A, B, S$ and T.

\section{Uniqueness:}

Assume $\mathrm{z}_{1}$ is second common fixed point.

Now assume $\mathrm{z} \neq \mathrm{z}_{1}$.

By considering $\mathrm{y}=\mathrm{z}_{1}, \mathrm{x}=\mathrm{z}$ in (3.1.4) we obtain

$F_{A z, B z_{1}, \text { a }}\left(t^{*}\right) \geq r F_{S z . T z_{1}, a}\left(t^{*}\right)$

$F_{z, z_{1}, \text { a }}\left(t^{*}\right) \geq r F_{z, z_{1}, a}\left(t^{*}\right)>F_{z, z_{1}, a}\left(t^{*}\right)$

$F_{z, z_{1}, a}\left(t^{*}\right)>F_{z . z_{1}}, a\left(t^{*}\right)$ which is absurd. Hence $\mathrm{z}=\mathrm{z}_{1}$.

As a result, four self- mappings $\mathrm{A}, \mathrm{B}, \mathrm{S}$, and $\mathrm{T}$ have the only one common fixed point.

Now we justify our theorem as under.

\subsection{Example}

Let us take $\mathrm{X}=[0, \pi]$ and each $t \in[0,1]$, define

$F_{u, v}(t)=\left\{\begin{array}{cl}\frac{t}{t+|\alpha-\beta|}, & t>0 \\ 0, & t=0\end{array} \quad\right.$ for all $\alpha, \beta$ in $X, t>0$.

Define mappings $P, S, T \& Q: X \rightarrow X$ as

$A(x)=B(x)=\left\{\begin{array}{l}2 e^{-\pi x}, x \in\left[0, \frac{\pi}{2}\right) \\ \pi-x, x \in\left[\frac{\pi}{2}, \pi\right]\end{array}\right.$

and $S(x)=T(x)=\left\{\begin{array}{c}2 e^{-\pi x^{2}}, x \in\left[0, \frac{\pi}{2}\right) \\ x, x \in\left[\frac{\pi}{2}, \pi\right]\end{array}\right.$

Now $\mathrm{A}(X)=\mathrm{B}(\mathrm{X})=[0,2]$ and $\mathrm{S}(\mathrm{X})=\mathrm{T}(\mathrm{X})=[0, \pi]$

implies $A(X) \subseteq T(X)$ and $B(X) \subseteq S(X)$.

Clearly $\frac{\pi}{2}$ and 1 are coincidence points for the mappings $\mathrm{B}, \mathrm{T}$.

At $\mathrm{x}=\frac{\pi}{2}, \mathrm{~B}\left(\frac{\pi}{2}\right)=\mathrm{T}\left(\frac{\pi}{2}\right)$ and $B T\left(\frac{\pi}{2}\right)=B\left(\frac{\pi}{2}\right)=\frac{\pi}{2}$,

$T B\left(\frac{\pi}{2}\right)=\mathrm{T}\left(\frac{\pi}{2}\right)=\frac{\pi}{2}$ implies $B T\left(\frac{\pi}{2}\right)=T B\left(\frac{\pi}{2}\right)$.

At $\mathrm{x}=1, \mathrm{~B}(1)=\mathrm{T}(1)$ and $\mathrm{BT}(1) \neq \mathrm{TB}(1)$.

Thus the pairs $(A, S),(B, T)$ satisfy OWC but are not weakly compatible.

If $x_{n}=\frac{\pi}{2}-\frac{1}{n}$ for all $\mathrm{n} \geq 1$. Then

$\mathrm{S} x_{n}=\mathrm{T} x_{n}=\mathrm{S}\left(\frac{\pi}{2}-\frac{1}{n}\right)=\frac{\pi}{2}-\frac{1}{n} \rightarrow \frac{\pi}{2}$.

$\mathrm{A} x_{n}=\mathrm{B} x_{n}=\mathrm{A}\left(\frac{\pi}{2}-\frac{1}{n} \quad\right)=\pi-\left(\frac{\pi}{2}-\frac{1}{n}\right)=\frac{\pi}{2}+\frac{1}{n} \quad \rightarrow \frac{\pi}{2}$ as $\mathrm{n} \rightarrow \infty$. 
$\Rightarrow \mathrm{A} x_{n}, \mathrm{~S} x_{n}, \mathrm{~T} x_{n}, \mathrm{~B} x_{n} \rightarrow \mathrm{S}\left(\frac{\pi}{2}\right)$ as $\mathrm{n} \rightarrow \infty$.

This gives the pairs of maps $(A, S),(B, T)$ sharing the CLR's property with OWC.

Thus A, B, S and T satisfy all the norms of Theorem and having the unique commonly fixed point at $\frac{\pi}{2}$ as $\mathrm{A}\left(\frac{\pi}{2}\right)=\mathrm{S}\left(\frac{\pi}{2}\right)=\mathrm{B}\left(\frac{\pi}{2}\right)=\mathrm{T}\left(\frac{\pi}{2}\right)=\frac{\pi}{2}$.

Now we present another generalization of Theorem (A) as under.

Theorem 3.3 Let A, B, S and T be self -mappings on a complete probabilistic 2-metric space

$\left(\widetilde{\mathrm{X}}, \mathrm{F}, t^{*}\right)$ satisfying :

(3.3.1) $\mathrm{A}(\widetilde{\mathrm{X}}) \subseteq \mathrm{T}(\widetilde{\mathrm{X}}), \mathrm{B}(\widetilde{\mathrm{X}}) \subseteq \mathrm{S}(\widetilde{\mathrm{X}})$

(3.3.2) the pair $(A, S)$ satisfies $C L R^{\prime}$ s property with OWC and (B, T) satisfies OWC.

(3.3.3) Further $F_{A x \text {. By, } a}\left(t^{*}\right) \geq \mathrm{r} F_{S x}$. Ty, $a\left(t^{*}\right)$ for all elements $\mathrm{x}, \mathrm{y}$ in $\widetilde{\mathrm{X}}$ and $t^{*}>0$

$\mathrm{r}$ is continuous self-map on $[0,1]$ such that $\mathrm{r}\left(t^{*}\right)>t^{*}$ for each $\mathrm{o}<t^{*}<1$.

Then $\mathrm{A}, \mathrm{B}, \mathrm{S}$ and $\mathrm{T}$ have unique common fixed point in $\widetilde{\mathrm{X}}$.

\section{Proof:}

Take the constructed sequences $\left\langle\mathrm{x}_{\mathrm{n}}\right\rangle,\left\langle\mathrm{y}_{\mathrm{n}}\right\rangle$ in Theorem (3.1) as

$\left\langle y_{2 n}\right\rangle=\mathrm{A} x_{2 n}=\mathrm{T} x_{2 n+1}$ and $\left\langle y_{2 n+1}\right\rangle=\mathrm{B} x_{2 n+1}=\mathrm{S} x_{2 n+2}$.

It is already shown that $\left\langle y_{n}\right\rangle$ as cauchy sequence.

As a result each sub sequence has the same limit point $\mathrm{z}$ in complete space $\widetilde{\mathrm{X}}$.

That is $\mathrm{Ax}_{2 \mathrm{n}}, \mathrm{Sx}_{2 \mathrm{n}} \rightarrow \mathrm{z}$ and $\mathrm{Bx}_{2 \mathrm{n}+1}, \mathrm{Tx}_{2 \mathrm{n}+1} \rightarrow \mathrm{z}$.

The pair $(\mathrm{A}, \mathrm{S})$ obeys CLRs-property this implies there is a sequence $\left\langle z_{n}\right\rangle$ such that $A z_{n}, S z_{n} \rightarrow S v$ for some $\mathrm{v}$ in $\widetilde{\mathrm{X}}$.

Claim $\mathrm{z}=\mathrm{Sv}$.

By putting $y=x_{2 n+1}, x=z_{n}$, in (3.3.3), that results

$F_{A z_{n}, B x_{2 n+1,}, a}\left(t^{*}\right) \geq r F_{S Z_{n} . T x_{2 n+1}, a}\left(t^{*}\right)$ as $\mathrm{n} \rightarrow \infty$

$\Rightarrow F_{S v, z, \text { a }}\left(t^{*}\right) \geq r F_{S v, z, a}\left(t^{*}\right)>F_{S v, z, a}\left(t^{*}\right)$,

$\Rightarrow F_{S v, z, a}\left(t^{*}\right)>F_{S v, z, a}\left(t^{*}\right)$.

This is absurd. As a result $\mathrm{Sv}=\mathrm{z}$.

Claim Av $=$ Sv.

By inserting $\mathrm{x}=\mathrm{v}, \mathrm{y}=\mathrm{x}_{2 \mathrm{n}+3}$ in (3.3.3), that results

$F_{A v, B x_{2 n+3, a}}\left(t^{*}\right) \geq r F_{S v} . T x_{2 n+3}, a\left(t^{*}\right)$ letting as $\mathrm{n} \rightarrow \infty$

$\Rightarrow F_{A v, z, \quad \text { a }}\left(t^{*}\right) \geq r F_{S v . z, a}\left(t^{*}\right)$ using (3.3.8)

$\Rightarrow F_{A v, s v, \text { a }}\left(t^{*}\right) \geq r F_{S v} . s v, a\left(t^{*}\right)=\mathrm{r}(1)=1$. 
$\Rightarrow \mathrm{Av}=\mathrm{Sv}=\mathrm{z}$

Since the pair $(A, S)$ satisfies OWC property, that results

$A v=S v \Rightarrow S A v=A S v$. This gives $A z=S z$.

Claim Az $=\mathrm{z}$.

By replacing $y=x_{2 n+1}, x=z$ in (3.3.3), as a result

$F_{A z, B x_{2 n+1,} \text { a }}\left(t^{*}\right) \geq r F_{S z . T x_{2 n+1}, a}\left(t^{*}\right)$. As $\mathrm{n} \rightarrow \infty$

$\Rightarrow F_{A z, z, \text { a }}\left(t^{*}\right) \geq r F_{S z, z, a}\left(t^{*}\right)$, using (3.3.14)

$\Rightarrow F_{A z, z, \quad \mathrm{a}}\left(t^{*}\right) \geq r F_{A z . z, a}\left(t^{*}\right)>F_{A z, z, a}\left(t^{*}\right)$,

$\Rightarrow F_{A z, z, \quad \mathrm{a}}\left(t^{*}\right)>F_{A z, z, a}\left(t^{*}\right)$.

This is a contradiction. Consequently $\mathrm{Az}=\mathrm{z}$.

By combining (3.3.14) and (3.3.18) gives $z=S z=A z$.

Since $\mathrm{Az} \in \mathrm{A}(\widetilde{\mathrm{X}}) \subseteq \mathrm{T}(\widetilde{\mathrm{X}})$ then $\exists \omega \in \widetilde{\mathrm{X}}$ such that $\mathrm{Az}=\mathrm{T} \omega$.

Claim $\mathrm{z}=\mathrm{B} \omega$.

By using $\mathrm{x}=\mathrm{x}_{4 \mathrm{n}}, \mathrm{y}=\omega$ of (3.3.4), we obtain

$F_{A x_{2 n,}, B \omega}\left(t^{*}\right) \geq r F_{S x_{2 n,} . T \omega, a}\left(t^{*}\right)$.

Taking limit as $\mathrm{n} \rightarrow \infty$ and from (3.3.19) and (3.3.20) we get

$\Rightarrow F_{z, B \omega}\left(t^{*}\right) \geq r F_{z, z, a}\left(t^{*}\right)=\mathrm{r}(1)=1$.

Thus $\mathrm{z}=\mathrm{B} \omega=\mathrm{T} \omega$.

Since the pair $(\mathrm{B}, \mathrm{T})$ obeys OWC property gives

$\mathrm{B} \omega=\mathrm{T} \omega \Rightarrow \mathrm{BT} \omega=\mathrm{TB} \omega$ implying $\mathrm{Bz}=\mathrm{Tz}$.

Claim $\mathrm{z}=\mathrm{Bz}$.

Applying $\mathrm{x}=\mathrm{y}=\mathrm{z}$ in (3.3.3), this resulting

$F_{A z, B z, \text { a }}\left(t^{*}\right) \geq r F_{S z . T z, a}\left(t^{*}\right)$,

using (3.3.19) and (3.3.24)

$\Rightarrow F_{z, \mathrm{Bz}, \quad \mathrm{a}}\left(t^{*}\right) \geq r F_{z . B z, a}\left(t^{*}\right)>F_{z . B z, a}\left(t^{*}\right)$,

$\Rightarrow F_{z, \mathrm{Bz}, \quad \mathrm{a}}\left(t^{*}\right)>F_{z, B z, a}\left(t^{*}\right)$.

Contradicting the fact implies $\mathrm{Bz}=\mathrm{z}$.

As a result $\mathrm{Az}=\mathrm{Bz}=\mathrm{Sz}=\mathrm{Tz}=\mathrm{z}$.

As a consequence four self-mappings $\mathrm{A}, \mathrm{B}, \mathrm{S}$, and $\mathrm{T}$, there is a fixed point commonly.

Uniqueness can be easily proved as in the Theorem (3.1). 
Now the Theorem (3.3) can be supported by discussing with suitable example.

\subsection{Example}

We choose $X=[0,1], d$ be usual metric on $X$ and each $t \in[0,1]$, define $F_{u, v}(\mathrm{t})=\left\{\begin{array}{cl}\frac{t}{t+|\alpha-\beta|}, & t>0 \\ 0, & t=0\end{array} \quad\right.$ for all $\alpha, \beta$ in $X, t>0$.

Choose mappings $P, S, T \& Q: X \rightarrow X$ as

$P(x)=Q(x)=\left\{\begin{array}{r}1-2 x, x \in[0,0.2] \\ x^{2}, x \in(0.2,1]\end{array}\right.$

and $S(x)=T(x)=\left\{\begin{array}{ll}3 x, & x \in[0,0.2] \\ x^{3}, & x \in(0.2,1]\end{array}\right.$.

Now $\mathrm{P}(X)=\mathrm{Q}(X)=(0.04,1]$ and $\mathrm{S}(\mathrm{X})=\mathrm{T}(X)=[0,1]$

so that $P(X) \subseteq T(X)$ and $Q(X) \subseteq S(X)$.

Clearly 0.2 and 1 are coincedence points of the graphs Q, T.

At $\mathrm{x}=0.2, \mathrm{Q}(0.2)=\mathrm{T}(0.2)=0.6$ but $\mathrm{QT}(0.2)=\mathrm{Q}(0.6)=0.36, \mathrm{TQ}(0.2)=\mathrm{T}(0.6)=0.216$

At $\mathrm{x}=1, \mathrm{Q}(1)=\mathrm{T}(1)$ and $Q T(1)=Q(1)=1=T(1)=T Q(1)$.

This demonstrates that the pairs $(\mathrm{P}, \mathrm{S}),(\mathrm{Q}, \mathrm{T})$ are OWC mappings, although they are not weakly compatible.

If we choose $x_{n}=1-\frac{4}{3 n}$ for all $\mathrm{n} \geq 1$. Then

$\mathrm{P} x_{n}=\mathrm{P}\left(1-\frac{4}{3 n}\right)=\left(1-\frac{4}{3 n}\right)^{2} \rightarrow 1$

$\mathrm{S} x_{n}=\mathrm{S}\left(1-\frac{4}{3 n}\right)=\left(1-\frac{4}{3 n}\right)^{3} \rightarrow 1$ as $\mathrm{n} \rightarrow \infty$.

This implies $\mathrm{P} x_{n}, \mathrm{~S} x_{n} \rightarrow \mathrm{S}(1)$ as $\mathrm{n} \rightarrow \infty$.

This gives the pair $(P, S)$ satisfies $C L R^{\prime}$ s-property with $O W C$ and the pair $(Q, T)$ is OWC.

Thus the mappings $\mathrm{P}, \mathrm{Q}, \mathrm{S}$ and T satisfy all the norms of the Theorem (3.3), containing unique common fixed point at 1 as $1=P(1)=Q(1)=S(1)=T(1)$.

\section{CONCLUSION}

In this paper Theorem (A) is generalized in two ways.

(a) Theorem (3.1) is formulated by employing CLR's-property and applying OWC for both the pairs instead of assuming weakly compatible mappings. 


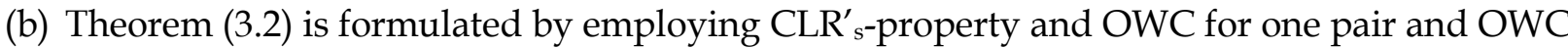
for the other pair instead of assuming weakly compatible mappings.

Further these two results are justified with suitable examples.

Conflicts of Interest: The author(s) declare that there are no conflicts of interest regarding the publication of this paper.

\section{REFERENCES}

[1] K. Menger, Statistical metrics, Proc. Natl. Acad. Sci. USA. 28(12) (1942) ,535-537.

[2] C. Alsina, B. Schweizer, and A. Sklar, On the definition of a probabilistic normed space, Aequationes Math. 46(1-2) (1993), 91-98.

[3] S. N. Mishra, N. Sharma and S. L. Singh, Common fixed points of maps on fuzzy Metric spaces, Int. J. Math. Math. Sci. 17(1994), 253-258.

[4] Altun, Ishak, and Duran Turkoglu, Some fixed point theorems for weakly compatible mappings satisfying an implicit relation, Taiwan. J. Math. 13 (2009), 1291-1304.

[5] X. Zhang, H. He, Y. Xu, A fuzzy logic system based on Schweizer-Sklar t-norm, Sci. China Ser. F: Inform. Sci. 49(2) (2006), 175-188.

[6] V. M. Sehgal, A. T. Bharucha-Reid, Fixed points of contraction mappings on probabilistic metric spaces, Math. Syst. Theory 6(1-2) (1972), 97-102.

[7] M. A. Al-Thagafi, N. Shahzad, A note on occasionally weakly compatible Maps, Int. J. Math. Anal 3.2 (2009), 55-58.

[8] V.K. Gupta, A. Jain and R. Kumar, Common fixed point theorem in probabilistic 2-Metric space by weak compatibility, Int. J. Theor. Appl. Sci. 11(1) (2019), 09-12.

[9] S. Chauhan, W. Sintunavarat, and P. Kumam, Common fixed point theorems for weakly compatible mappings in fuzzy metric spaces using (JCLR) property, Appl. Math. 3 (2012), 976-982.

[10]K. Satyanna, V. Srinvas, Fixed point theorem using semi compatible and sub sequentially continuous mappings in Menger space, J. Math. Comput. Sci. 10(6) (2020), 2503-2515. 